\title{
MCT1 confirmed in rat striated muscle mitochondria
}

\author{
C. Eric Butz, ${ }^{\mathbf{1}}$ Grant B. McClelland, ${ }^{\mathbf{1 , 2}}$ and George A. Brooks ${ }^{\mathbf{1}}$ \\ ${ }^{1}$ Department of Integrative Biology, University of California, Berkeley, California 94720; \\ and ${ }^{2}$ Department of Biology, McMaster University, Hamilton, Ontario L8S 4K1, Canada
}

Submitted 6 January 2004; accepted in final form 27 April 2004

Butz, C. Eric, Grant B. McClelland, and George A. Brooks. MCT1 confirmed in rat striated muscle mitochondria. J Appl Physiol 97: 1059-1066, 2004. First published April 30, 2004; 10.1152/ japplphysiol.00009.2004.-We sought to test the hypothesis that monocarboxylate transporter isoform 1 (MCT1) is the inner mitochondrial membrane lactate/pyruvate transporter, and, as such, contributes to functioning of the intracellular lactate shuttle. However, presence of a mammalian mitochondrially localized MCT1 (mMCT1) has been contested. We sought to confirm by Western blotting the mitochondrial localization of MCT1 in rat cardiac, soleus, and extensor digitorum longus muscles utilizing three different cell fractionation methods and three different antibodies. We performed Western blotting using antibodies to cell membrane glucose transporter isoform GLUT1, inner mitochondrial constituent cytochrome oxidase, the monocarboxylate transporter protein chaperone CD147, as well as custom and commercially available MCT1 antibodies. Western blots demonstrated similar results with each MCT1 antibody and two of three methods of fractionation. MCT1 was found in the mitochondria, as well as in the sarcolemmal membrane and whole muscle homogenates. Probing with GLUT1 and CD147 demonstrated that mitochondrial fractions were not contaminated with sarcolemmal remnants. Probing with cytochrome oxidase showed mitochondrial localization of MCT1. Comparison of these results to the findings of others indicates that the most likely source of discrepancy is the cell fractionation procedure utilized.

lactate; lactate shuttle; monocarboxylate transporter; monocarboxylate transporter isoform 1

LACTATE FORMED IN THE CYTOSOL of working muscles can be imported to the mitochondrial reticulum (18) for oxidation or exported from the cell; exported lactate can be taken up by adjacent muscle fibers or anatomically remote tissues for oxidation or gluconeogenesis involving a "cell-cell lactate shuttle" (5). Transport of lactate across lipid bilayer membranes is facilitated by a family of transporter proteins termed monocarboxylate transporters (MCT), first described by Garcia et al. (15). Since initial discovery, a family of eight MCTs have been characterized $(14,15,25,30)$ that exhibit tissue and cell domain specificities as well as interspecies distribution differences. Isoforms MCT1 and MCT4 have been found in abundance in striated muscle homogenates $(3,6)$. Pellerin et al. (23) found MCT1 and MCT2 in mouse brain and proposed an astrocyte-neuron lactate shuttle. McClelland et al. (21) demonstrated existence of MCT2 in the rat liver peroxisomes and ascribed its functionality to an intracellular redox shuttle system, and MCT3 is localized in retina (30).

The intracellular lactate shuttle (ILS) was proposed (6) to specify how the products of glycolysis, pyruvate and NADH,

\footnotetext{
Address for reprint requests and other correspondence: G. A. Brooks, Exercise Physiology Laboratory, Dept. of Integrative Biology, 5101 Valley Life Science Bldg., University of California, Berkeley, CA 94720-3140 (Email: gbrooks@socrates.berkeley.edu).
}

in the form of lactate molecules, are moved into mitochondria for oxidation. For red striated $(5,6)$ and cardiac muscle $(10)$, the ILS is likely the predominant pathway for carbon and reducing equivalent flux. In the process, cytosolic $\mathrm{NAD}^{+}$is regenerated, allowing glycolysis to continue. Although pyruvate can be transported into the mitochondria, most of the cytosolic pyruvate is reduced to lactate (10-fold greater concentration at rest, over 100 times greater during higher rates of energy flux), making lactate the numerically more consequential cytosolic organic anion of the two. Localization of MCT1 to the mitochondrial membrane was initially demonstrated by both Western blotting (utilizing a cell fractionation process giving both subsarcolemmal and interfibrillar mitochondria) and electron microscopic techniques in rats (6). Subsequently, by use of a different cell fractionation process, Western blotting demonstrated mitochondrial MCT1 (mMCT1) and mitochondrial lactate dehydrogenase in humans (12) and rats (20).

In contrast, Bonen et al. (3) utilized a different cell fractionation technique but failed to find mitochondrially localized MCT1. This cell fractionation technique (11) provides a lithium bromide (LiBr)-treated fraction that yields an internal membrane (L-IM) fraction that contains a pool of GLUT4 vesicles translocated to the sarcolemma and $\mathrm{T}$ tubules as a result of contraction due to an acute bout of exercise (27) or electrical stimulation (26). These studies $(11,26,27)$ have provided valuable information about this glucose transporter, but GLUT4 is not found in the mitochondrion, and the L-IM fraction was never purported to be representative of the mitochondrion.

Although the preceding fractionation procedure was used to investigate skeletal muscle mitochondria, other tissues and methods have been employed as well. Data have been published indicating little MCT1 in the pancreas and no MCT1 in the mitochondrion of the $\beta$-islets (31). Furthermore, the authors went on to state, "Indeed, we have not detected any MCT1 associated with mitochondria in any cell type or in isolated mitochondrial preparations" (Ref. 31, p. 64). Similarly, attempts with electron microscopy failed to find evidence of mitochondrial localization above background levels in cardiac sarcolemma or mitochondria (16). Unfortunately, there were no descriptions of how background levels were evaluated, no data, and no explanation for the failure to visualize sarcolemmal MCT1. Furthermore, those results $(16,31)$ are in contrast to those of Valenti et al. (29), who demonstrated a functional ILS in rat heart mitochondria. Valenti et al., however, interpreted this to be an export of pyruvate out of the mitochondrion.

In view of the importance of a mitochondrial location of MCT1 to the ILS hypothesis and controversies over the exis-

\footnotetext{
The costs of publication of this article were defrayed in part by the payment of page charges. The article must therefore be hereby marked "advertisement" in accordance with 18 U.S.C. Section 1734 solely to indicate this fact.
} 
tence of mMCT1 and the ILS, we sought to test the hypothesis that MCT1 is the inner mitochondrial membrane lactate/pyruvate transporter and, as such, contributes to functioning of the ILS. Therefore, we probed tissue homogenates and cell fractions using three different fractionation procedures and three different MCT1 antibodies (raised in two different hosts). To establish cellular locations of MCT1 in situ, we also probed for proteins whose locations are now well accepted; these include the constitutive sarcolemmal glucose transporter (GLUT1), the inner mitochondrial membrane component cytochrome oxidase (COX), and the MCT chaperone CD147. Results point toward differences in membrane separation procedures as the source of discrepancy.

\section{METHODS}

\section{Animal Care}

Female Sprague-Dawley rats were used throughout. Animals were maintained at $25^{\circ} \mathrm{C}$ on a 12:12-h light-dark cycle with access to standard rodent laboratory chow and water ad libitum. All procedures were in accordance with accepted protocols of the Animal Care and Use Committee at the University of California, Berkeley (AUP no. R017-1000R).

\section{Cellular Fractionation}

Comparisons of three different fractionation procedures were performed. These procedures (hereafter identified to as methods 1,2, and 3) are described below.
Subcellular fractionation procedure (method 1). Whole muscle homogenates (MU), sarcolemmal fractions (SL), and mitochondrial fractions (MI) were isolated as previously described (12); these procedures are shown in Fig. 1. Briefly, animals were euthanized by pentobarbital overdose. Heart, soleus, and extensor digitorum longus (EDL) were quickly removed and frozen. Approximately $200 \mathrm{mg}$ of tissue were homogenized in ice-cold solution A (210 mM sucrose, 2 mM EGTA, $40 \mathrm{mM} \mathrm{NaCl}$, and $30 \mathrm{mM}$ HEPES, $\mathrm{pH}$ 7.4) by use of a motor-driven glass homogenizer. The homogenate was centrifuged at $600 \mathrm{~g}$ in a Sorvall RC-5 Superspeed centrifuge for $10 \mathrm{~min}$ at $4{ }^{\circ} \mathrm{C}$ to eliminate erythrocyte material. One milliliter of supernatant was diluted with $0.75 \mathrm{ml}$ of buffer $B(1.167 \mathrm{M} \mathrm{KCl}$ and $58.3 \mathrm{mM}$ $\mathrm{Na}_{4} \mathrm{P}_{2} \mathrm{O}_{7} \cdot 10 \mathrm{H}_{2} \mathrm{O}$, pH 7.4) and centrifuged in a Beckman L8-7M ultracentrifuge at $230,000 \mathrm{~g}$ for $120 \mathrm{~min}$ at $4{ }^{\circ} \mathrm{C}$ to remove contractile proteins. The pellet was washed in $1 \mathrm{ml}$ of buffer $C$ ( $1 \mathrm{mM}$ EDTA and $10 \mathrm{mM}$ Tris, $\mathrm{pH}$ 7.4). The pellet was resuspended in $200 \mu \mathrm{l}$ of buffer $C$ and $66 \mu$ of $16 \%$ SDS and centrifuged at room temperature for 20 min to remove any insoluble materials. Supernatants containing crude MU were aliquoted and stored at $-80^{\circ} \mathrm{C}$. Unless otherwise stated, the Sorvall and Beckman refrigerated centrifuges were used for all subsequently described procedures.

The remaining supernatant of the $600 \mathrm{~g}$ spin was centrifuged at $10,000 \mathrm{~g}$ for $20 \mathrm{~min}$ at $4^{\circ} \mathrm{C}$. The supernatant from this spin was diluted with 0.75 volumes of buffer $B$ and centrifuged at 230,000 $g$ for 120 min. The pellet was washed in $1 \mathrm{ml}$ of buffer $C$ and then resuspended in $200 \mu \mathrm{l}$ of buffer $C$ and $66 \mu \mathrm{l}$ of $16 \%$ SDS and centrifuged at room temperature for $20 \mathrm{~min}$ to remove any insoluble materials. Supernatants containing SL were aliquoted and stored at $-80^{\circ} \mathrm{C}$.
Fig. 1. Flowchart for differential centrifugation procedure used in method 1. Note that mitochondrial fraction (MI) is pelleted in a $10,000 \mathrm{~g}$ step. MU, whole muscle homogenate fraction; SL, sarcolemmal fraction. See METHODS for details of procedure.

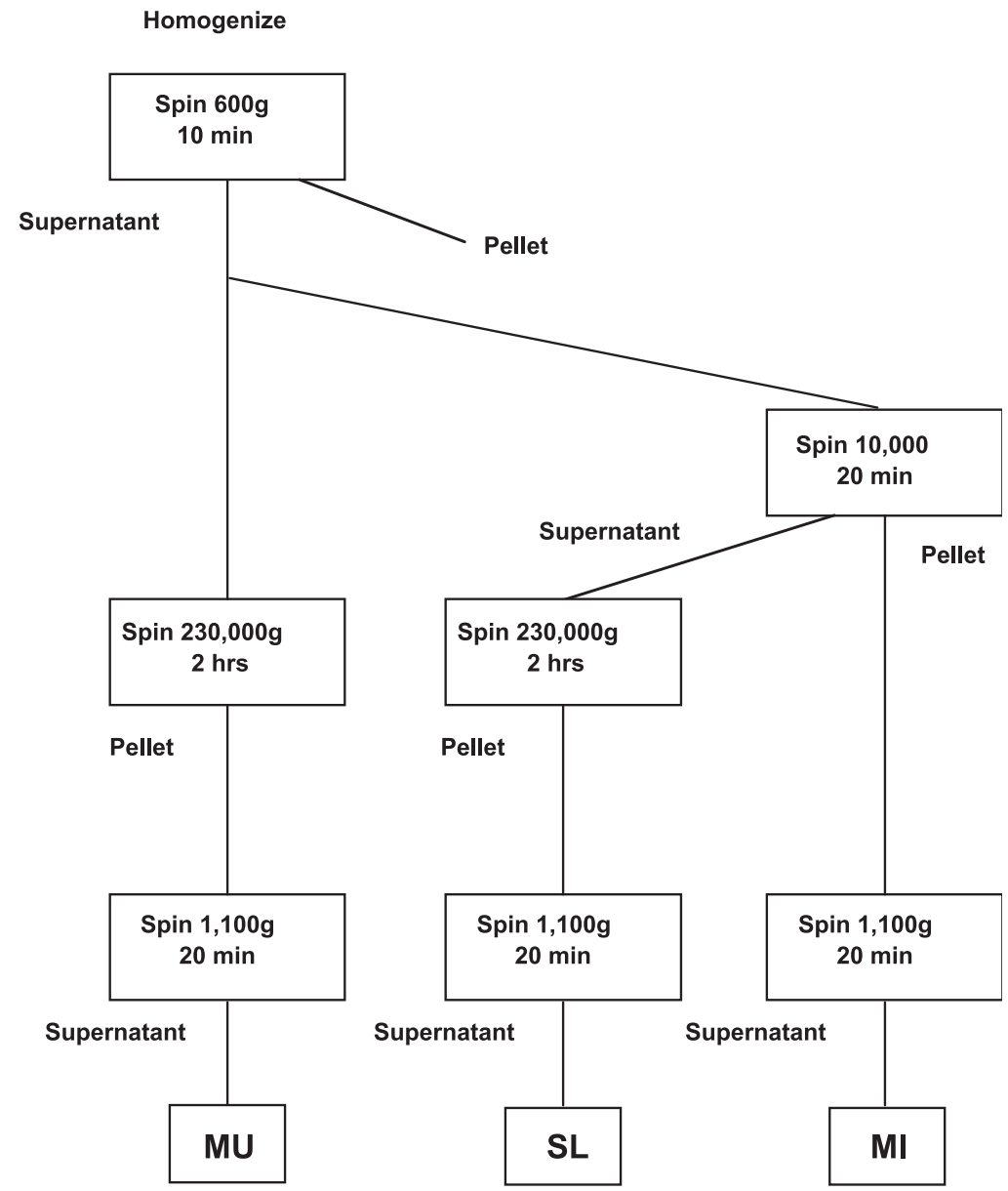


The pellet of the $10,000 \mathrm{~g}$ spin was washed in $1 \mathrm{ml}$ of buffer $C$ and then resuspended in $200 \mu \mathrm{l}$ of buffer $C$ and $66 \mu \mathrm{l}$ of $16 \%$ SDS and centrifuged at room temperature for $20 \mathrm{~min}$ to remove insoluble materials. Supernatants containing MI were aliquoted and stored at $-80^{\circ} \mathrm{C}$.

Isolation of mitochondrial populations (method 2). Subsarcolemmal (SM) and interfibrillar (IM) mitochondrial population were isolated as previously described by Bizeau et al. (2), as modified by Brooks et al. (6) and shown in Fig. 2. Briefly, animals were euthanized by pentobarbital overdose. Heart, soleus, and EDL were quickly removed and frozen. Tissue was weighed and immersed in ice-cold solution $1(100 \mathrm{mM} \mathrm{KCl}, 40 \mathrm{mM}$ Tris $\cdot \mathrm{HCl}, 10 \mathrm{mM}$ Tris base, $5 \mathrm{mM}$ $\mathrm{MgCl}_{2}, 1 \mathrm{mM}$ EDTA, and $1 \mathrm{mM}$ ATP, $\mathrm{pH}$ 7.5). Minced tissue was suspended in 10-fold (wt/vol) solution 1 and homogenized in an Ultra-Turrex (Cincinnati, OH) tissue homogenizer at $40 \%$ power for $10 \mathrm{~s}$. For this and all other procedures, homogenization was performed in an ice-ice water chilled vessel in a $4^{\circ} \mathrm{C}$ cloakroom. The homogenate was centrifuged at $800 \mathrm{~g}$ for $10 \mathrm{~min}$ in a refrigerated centrifuge (Tomy, model MRX-151). The pellet was used to prepare IM, as described below. The supernatant was filtered through a double layer of cheesecloth and centrifuged at $9,000 \mathrm{~g}$ for $10 \mathrm{~min}$ to pellet SM population. The supernatant was used to isolate sarcolemmal and remaining nonmitochondrial membranes. The SM pellet was resuspended in solution $2(100 \mathrm{mM} \mathrm{KCl}, 10 \mathrm{mM}$ Tris $\cdot \mathrm{HCl}, 10 \mathrm{mM}$ Tris

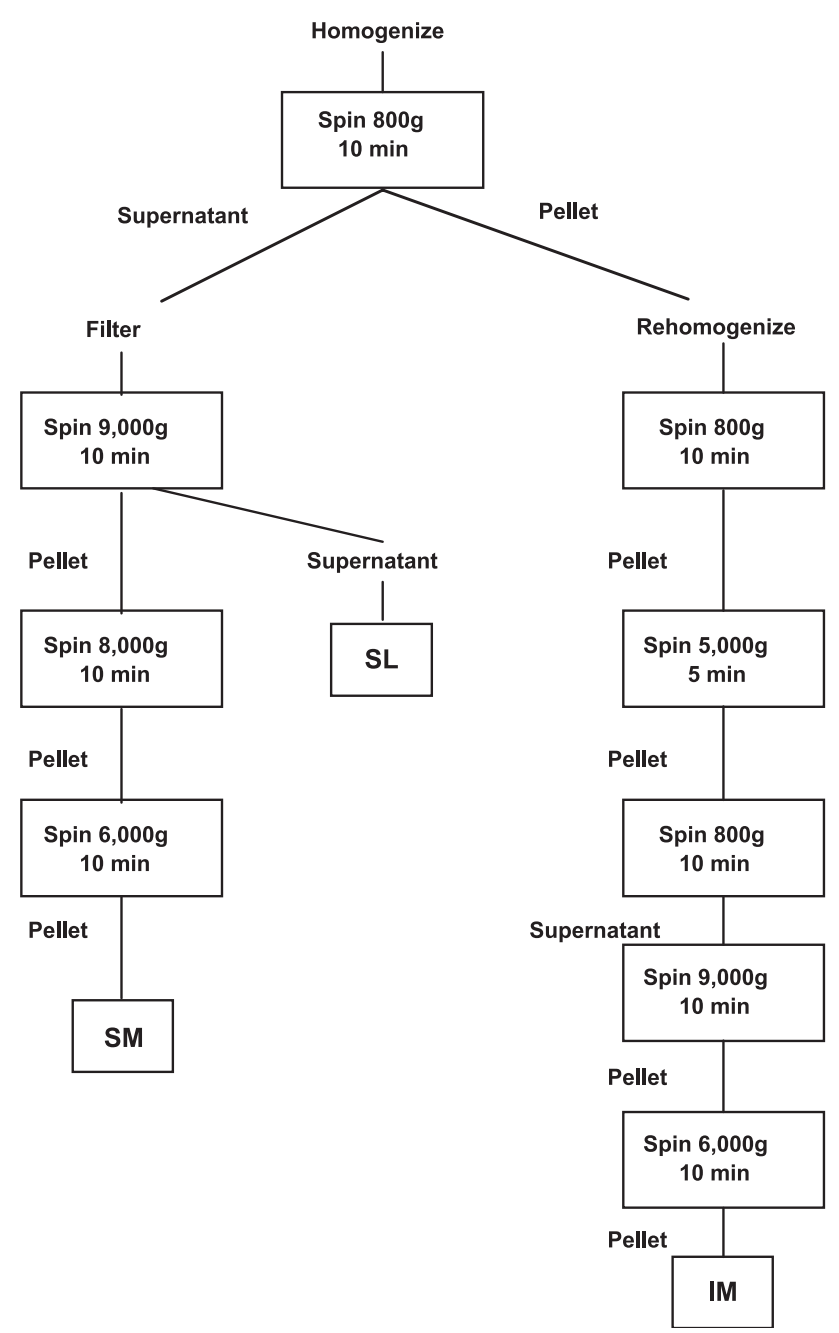

Fig. 2. Flowchart for differential centrifugation procedure used in method 2. Note that both subsarcolemmal (SM) and interfibrillar (IM) mitochondrial fractions are pelleted in 9,000 $g$ steps. See METHODS for details of procedure. base, $1 \mathrm{mM} \mathrm{MgSO}$, $0.1 \mathrm{mM}$ EDTA, $0.02 \mathrm{mM}$ ATP, and 1.5\% BSA, $\mathrm{pH} 7.4$ ) and centrifuged at $8,000 \mathrm{~g}$ for $10 \mathrm{~min}$. The supernatant was discarded and the pellet was resuspended in solution $2(100 \mathrm{mM} \mathrm{KCl}$, $10 \mathrm{mM}$ Tris $\cdot \mathrm{HCl}, 10 \mathrm{mM}$ Tris base, $1 \mathrm{mM} \mathrm{MgSO}$, $0.1 \mathrm{mM}$ EDTA, and $0.02 \mathrm{mM}$ ATP, $\mathrm{pH} 7.4$ ) and centrifuged at $6,000 \mathrm{~g}$ for $10 \mathrm{~min}$. The supernatant was discarded, and the pellet was resuspended in a small volume (1.0 ml, heart; $0.5 \mathrm{ml}$, soleus and EDL) of suspension buffer $(220 \mathrm{mM}$ sucrose, $70 \mathrm{mM}$ mannitol, $10 \mathrm{mM}$ Tris $\cdot \mathrm{HCl}$, and $1 \mathrm{mM}$ EDTA, pH 7.4).

The IM were isolated without proteolytic digestion by use of nagarse or trypsin because these proteolytic enzymes are known to degrade MCT1 and other inner membrane transport proteins (4). For preparation of IM populations, the IM pellet from the first $800 \mathrm{~g}$ spin was resuspended in a 10-fold dilution of solution 1 with a glass Teflon homogenizer, homogenized further in the Ultra-Turrex for $10 \mathrm{~s}$, and centrifuged at $800 \mathrm{~g}$ for $10 \mathrm{~min}$. The supernatant was discarded and the pellet was resuspended in a 10-fold dilution of solution 1 and centrifuged at 5,000 $\mathrm{g}$ for $5 \mathrm{~min}$. The supernatant was discarded, and the pellet was resuspended in 10-fold dilution of solution 2 and centrifuged at $800 \mathrm{~g}$ for $10 \mathrm{~min}$. The pellet was discarded, and the supernatant was centrifuged at $9,000 \mathrm{~g}$ for $10 \mathrm{~min}$. The supernatant was discarded, and the pellet was resuspended in 10-fold dilution of solution 3 and centrifuged at $6,000 \mathrm{~g}$ for $10 \mathrm{~min}$. The supernatant was discarded, and the pellet was resuspended in a small volume $(1.0 \mathrm{ml}$, heart; $0.5 \mathrm{ml}$, soleus and EDL) of suspension buffer.

Dombrowski fractionation procedure (method 3). Sarcolemmal membranes, $\mathrm{T}$ tubules, and intracellular membranes were isolated as described in Dombrowski et al. (11) and shown in Fig. 3. Hindlimb muscles were harvested and frozen for subsequent cell fractionation. All subsequent steps were performed at $4^{\circ} \mathrm{C}$ in a coldroom or in an ice-chilled vessel. Muscle was weighed and minced for $5 \mathrm{~min}$ in buffer $\alpha\left(10 \mathrm{mM} \mathrm{NaHCO}, 0.25 \mathrm{M}\right.$ sucrose, $5 \mathrm{mM} \mathrm{NaN}_{3}$, and $100 \mu \mathrm{M}$ PMSF, pH 7.0), $1 \mathrm{~g} / 15 \mathrm{ml}$ dilution. Minced muscle was homogenized using an Ultra-Turrex, twice at $5 \mathrm{~s}$ each time. An aliquot representing the whole muscle homogenate was saved. The homogenate was centrifuged at $1,300 \mathrm{~g}$ for $10 \mathrm{~min}$. The membrane pellet was resuspended in $2 \mathrm{~g} / 15 \mathrm{ml}$ dilution of buffer $\alpha$, rehomogenized, and again centrifuged at $1,300 \mathrm{~g}$ for $10 \mathrm{~min}$. The pellet was resuspended in buffer $\beta(0.5 \mathrm{M} \mathrm{LiBr}$ and $50 \mathrm{mM}$ Tris, $\mathrm{pH} 8.5)(1 \mathrm{~g}$ tissue $/ 25 \mathrm{ml}$ buffer) and stirred for $4 \mathrm{~h}$, providing the LiBr-treated membranes fraction PF1. The fraction was then centrifuged at 1,200 $\mathrm{g}$ for $5 \mathrm{~min}$, with the pellet representing fraction PF2. The supernatant was then centrifuged at $10,000 \mathrm{~g}$ for $30 \mathrm{~min}$ (pellet representing PF3) and then $53,000 \mathrm{~g}$ for $1 \mathrm{~h}$. The resulting pellet (PF4) was resuspended in buffer $\gamma(0.15 \mathrm{M} \mathrm{KCl}, 5 \mathrm{mM} \mathrm{MgSO}$, and $20 \mathrm{mM}$ HEPES, $\mathrm{pH}$ 6.8). The supernatant of the $53,000 \mathrm{~g}$ spin was centrifuged at $190,000 \mathrm{~g}$ for $1 \mathrm{~h}$, and the pellet (PF8) was then resuspended in buffer $\alpha$. The resuspended PF4 fraction was applied to 10,27 , and $35 \%$ discontinuous sucrose gradients (wt/wt), and the resuspended PF8 fraction was applied to 10 and $40 \%$ discontinuous sucrose gradients (wt/wt). Both gradients were centrifuged at $150,000 \mathrm{~g}$ for $16 \mathrm{~h}$. For the PF4 gradients, membranes at the $10 \% / 27 \%$ (PF5) and $27 \% / 35 \%$ (PF6) interfaces as well as the pellet (PF7) were recovered. For the PF8 gradients, membranes at the $10 \% / 40 \%$ (PF9) interface as well as the pellet (PF10) were recovered. All of these interfaces and pellets were diluted in sucrose-free buffer $\alpha$ and centrifuged at 190,000 $\mathrm{g}$ for $1 \mathrm{~h}$. Pellets were recovered and resuspended in buffer $\alpha$ and stored at $-80^{\circ} \mathrm{C}$ for Western blot analysis. For this path, PF6 represented T tubules and PF9 represented L-IM (LiBr-treated internal membranes fraction).

The supernatants recovered from the two $1,300 \mathrm{~g}$ spins were combined and centrifuged at $9,000 \mathrm{~g}$ for $10 \mathrm{~min}$. The supernatant of this spin was centrifuged for $1 \mathrm{~h}$ at $190,000 \mathrm{~g}$. The pellet was resuspended in buffer $\alpha$ and applied on discontinuous sucrose gradients $(25,32$, and $35 \% \mathrm{wt} / \mathrm{wt})$ and centrifuged at $150,000 \mathrm{~g}$ for $16 \mathrm{~h}$. The membranes at the sample/25\% (F3), 25\%/32\% (F4), and 32\%/ $35 \%$ (F5) interfaces as well as the pellet (F6) were recovered and 
Fig. 3. Flowchart for differential centrifugation procedure used in method 3. Note that mitochondria are likely pelleted in $13,000 \mathrm{~g}$ (PF3) and 53,000 $g$ (PF4) steps. Mitochondria are not likely to be found in supernatant from which the lithium bromide ( $\mathrm{LiBr}$ )-treated fraction that yields internal membranes (L-IM) fraction (PF9) is drawn. See METHODs for details of procedure.

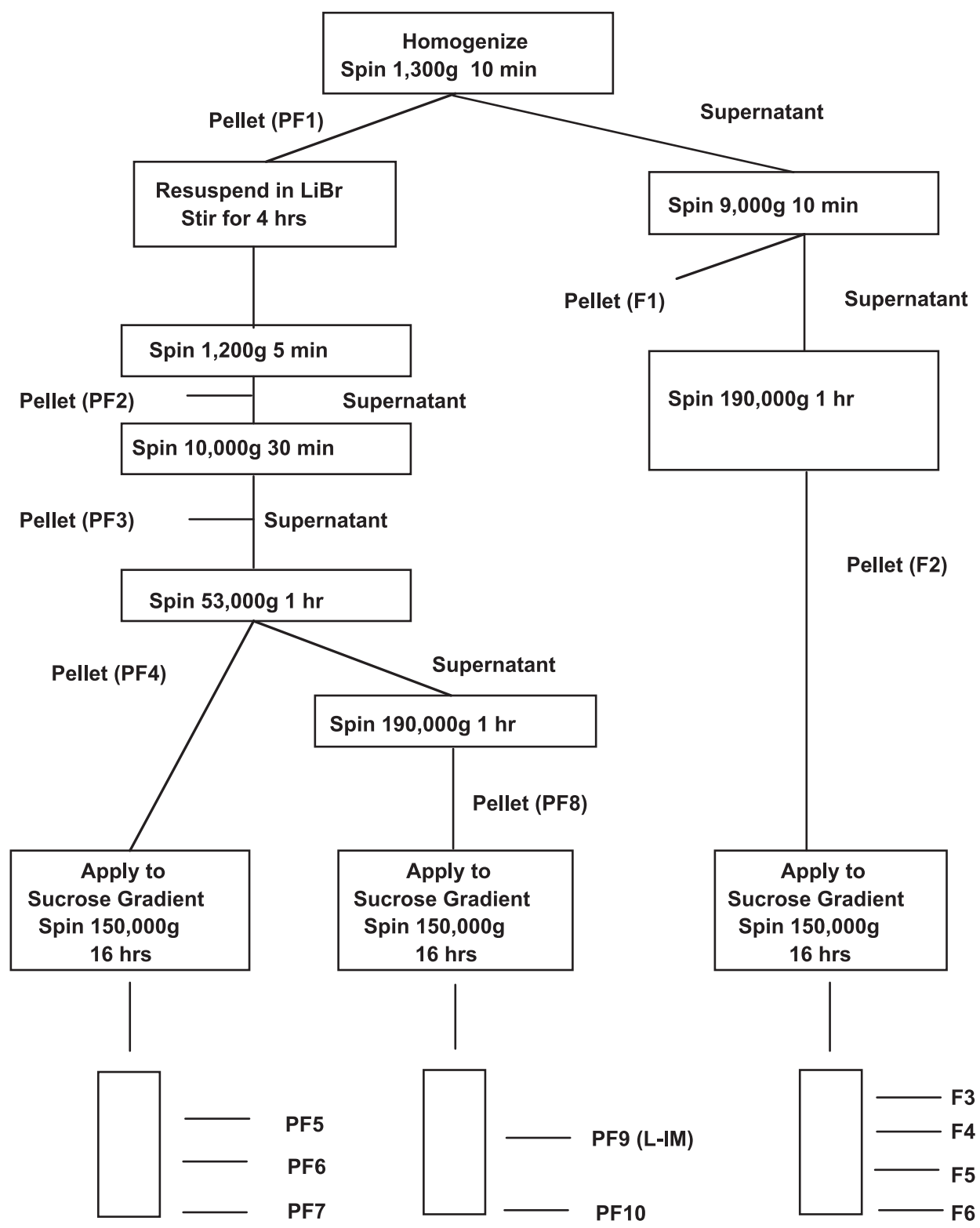

MU, SL, MI, SM, and IM fractions were probed with three different antibodies to rat MCT1. These anti-MCT1 antibodies were Brooks (custom, rabbit anti-rat), ADI (Alpha Diagnostics, rabbit anti-rat), and CHEM (Chemicon, chicken anti-rat). As described in

Table 1. Comparison of MCT1 antibodies

\begin{tabular}{|c|c|c|c|}
\hline Source & Sequence & Host & Reference \\
\hline GenBank & $\mathrm{N}^{\prime}-\mathrm{APLQNSS} G D P A E E E S P V-\mathrm{C}^{\prime}$ & & $\begin{array}{l}\text { GenBank } \\
\text { sequence }\end{array}$ \\
\hline Brooks & $\mathrm{N}^{\prime}-\underline{C} P L Q N S S G D P A E E E S P V-C^{\prime}$ & Rabbit & 7 \\
\hline $\begin{array}{l}\text { Alpha } \\
\text { Diagnostics }\end{array}$ & Proprietary & Rabbit & Proprietary \\
\hline Chemicon & 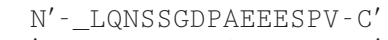 & Chicken & Data sheet \\
\hline Halestrap* & $\mathrm{N}^{\prime}-\underline{C} \mathrm{P} \underline{Q} Q N S$ S GDPAEEE SPV - C' & Rabbit & 24 \\
\hline
\end{tabular}

MCT1, monocarboxylate transporter 1. *Not used in this study but in Refs. 3 and 31. Antibody was developed from Chinese hamster ovary cells (24) and showed high interspecies homology in this $\mathrm{COOH}$-terminal portion of 2 mammalian species. 


\section{$45 \mathrm{kDa}$ \\ A}

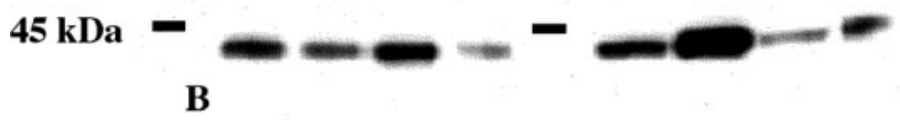

45 kDa
ADI

Chem
Fig. 4. Comparison of monocarboxylate transporter isoform 1 (MCT1) antibodies, using method 1. Shown are MU, SL, MI, and standard erythrocyte ghost membrane (Std) fractions for rat heart (left) and soleus (right). The same membrane was probed with Brooks custom antibody (A), Alpha Diagnostics (ADI) antibody $(B)$, and Chemicon (Chem) antibody $(C)$. See legends to Figs. 1-3 and METHODS for details.
C

Heart
Soleus
Table 1, like the sequence used by Halestrap and associates (24), antibodies to MCT1 are similar and target an immunoreactive portion of the MCT1 COOH terminal. Evaluation of mitochondrial fraction contamination by sarcolemma was performed by probing the membranes with anti-GLUT1 (Chemicon) antibody. A secondary confirmation of the lack of sarcolemmal contamination was performed by probing with an anti-CD147 antibody, a chaperone protein for MCT1 found only in the sarcolemma. Rat erythrocyte ghosts were used as a positive control for MCT1 and a negative control for GLUT1. Mitochondrial content was determined by probing membranes with antiCOX. Western blot probing was determined by enhanced chemiluminescence (NEN Life Sciences) and scanned with a Bio-Rad GS-700 laser densitometer, and, where appropriate, bands were quantified by software analysis using Molecular Analyst (Bio-Rad Laboratories). When membranes were used multiple times, they were stripped using Western Blot Recycling Kit (Alpha Diagnostics International) and washed in TTBS. Preliminary studies were performed to verify that previous antibody was completely removed by this method.

\section{Statistical Analysis}

Differences between antibodies were determined with the use of three animals each for heart and soleus, with each membrane probed sequentially with the three different MCT1 antibodies. Different membranes and antibodies were normalized by using the Std as 1.0 and are reported as means \pm SD. Quantified bands were compared by one-factor ANOVA. Significance was determined at the level of $P<$ 0.05 throughout.

\section{RESULTS}

Comparison of antibodies was performed by using cell fractionation method 1 and provided similar results for all three antibodies used (Fig. 4). For both heart and soleus muscle, MCT1 was found in the SL and the MU fractions. This is expected because the sarcolemma is a component of the whole muscle. Note also that the MI fraction was labeled by all three MCT1 antibodies. As expected, the Std (rat erythrocyte ghosts) was labeled, acting as a positive control. The heart MCT1 concentration was approximately the same in MU, SL, and MI fractions $(1.704 \pm 0.132,1.604 \pm 0.137$, and $1.669 \pm 0.301$, respectively), given equal mass of total protein per lane. For the soleus, MCT1 concentrations were greatest in the SL at more than twice the concentration of the MU fraction and seven times the concentration of the MI fraction (2.296 \pm $0.441,0.949 \pm 0.108$, and $0.327 \pm 0.098$, respectively).
Evaluation of fraction contamination for method 1 is shown in Fig. 5. The same polyvinylidene difluoride membrane was sequentially probed with MCT1, GLUT1, and CD147. Although the MI fraction binds MCT1, it does not show GLUT1 labeling. Here, the Std was not labeled with GLUT1, acting as a negative control. No CD147 was found in the MI fraction, but it was found in MU, SL, and Std as expected. For brevity, we do not show a version of Fig. 5 to demonstrate purity of membrane fractions obtained by method 2; such results have been previously demonstrated $(12,20)$.

To evaluate whether different fractionation procedures gave rise to idiosyncratic results, muscle separated by the three methods was compared. Figures 4 and 6 demonstrate that mitochondria from method 1 (MI) and method 2 (SM and IM) are labeled by MCT1. In Fig. 7, the L-IM fraction (PF9) from method 3 fails to demonstrate significant MCT1 labeling. It should be noted that the same membrane failed to show label for COX in PF9 (the L-IM fraction purported to represent

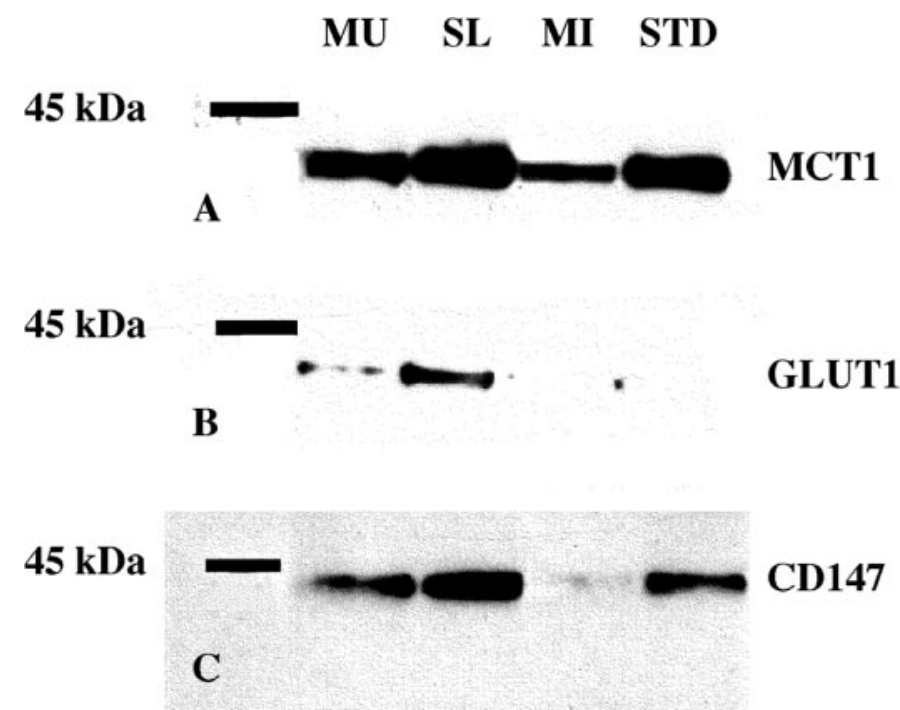

Fig. 5. Lack of cross contamination of fractions due to fractionation process (using method 1). Shown are MU, SL, MI, and Std fractions for rat soleus probed with MCT1 antibody (A), GLUT1 antibody $(B)$, and CD147 antibody (C). Similar results obtained for method 2; data not shown. See legends to Figs. 1-3 and METHODS for details. 


\section{SM IM SL STD \\ $45 \mathrm{kDa}$

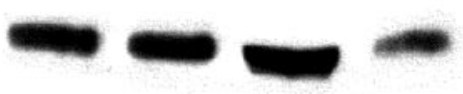

Fig. 6. MCT1 is found in all mitochondrial populations (using method 2). Fractions for rat heart were probed with MCT1 antibody. See legends to Figs. $1-3$ and METHODS for details.

mitochondria), indicating a low mitochondrial content. In fact, by method 3, MCT1 and COX labeling were found dispersed into many other fractions (F1, F2, F6, PF1, PF2, PF3, and PF4), including a rather large amount in the PF3 fraction. These results indicate that much of the mitochondrial mass was removed before the sucrose gradient centrifugation step and never reached the PF9 fraction. Also note that the Std was labeled with MCT1 but not COX, demonstrating the lack of mitochondrial labeling when inner mitochondrial membrane debris were not present.

Because the $\mathrm{LiBr}$ step was considered a possible source of the absence of mitochondrial remnants in the PF9 fraction, method 3 was performed both with and without the $\mathrm{LiBr}$ digestion. The results indicate that similar protein concentrations and similar fraction contents were recovered in each fraction, with and without $\mathrm{LiBr}$ digestion (data not shown). The only apparent difference was that the total yield was much less when $\mathrm{LiBr}$ was not used.

\section{DISCUSSION}

We confirm the existence of MCT1 in the mitochondria of rat skeletal and cardiac muscle. Results with three antibodies raised in two different hosts and two of the three different fractionation procedures provide evidence that MCT1 is found not only in the sarcolemma but also in the subsarcolemmal and interfibrillar mitochondrial fractions. Probing with GLUT1 and CD147 demonstrates that there was no significant contamination of the mitochondrial fractions by the sarcolemma. All three antibodies targeted MCT1, but fractionation procedures yielded variable results. Hence, we reproduce our results as well as those of others; what differs is the interpretation.
As has been previously discussed, the Brooks MCT1 antibody, raised against the $\mathrm{COOH}$ terminal of the rat MCT1, has low homology with other known MCT sequences and has no significant homology with any protein other than MCT1 (6). In fact, review of the published sequences used to develop the various antibodies (see Table 1) indicates that all the MCT1 antibodies targeted the same section of the $\mathrm{COOH}$ terminal of the rat MCT1. Because these antibodies were raised in either the rabbit (Brooks and ADI) or chicken (Chemicon), it is unlikely that there have been host-specific problems in MCT1 identification. One possible consideration is that Bonen et al. (3) and Zhao et al. (31) used antibodies developed from Chinese hamster ovary cells, but there is extremely high cross-species homology in the immunoreactive section of the MCT1 COOH terminal used by many investigators to develop antibodies to MCT1. However, as asserted by Zhao et al., the Chinese hamster ovary-derived antibody to MCT1 readily reacts across cells and tissues taken or derived from a variety of mammalian species.

The possibility that monocarboxylates are transported into the mitochondria via an as yet undetermined, six-transmembrane (6TM) transporter was considered. The family of 6TM transporters has been investigated, and a putative, $30-\mathrm{kDa}$ pyruvate transporter was partially purified $(9,22)$. However, the protein we found in mitochondrial and sarcolemmal membranes was $\sim 43 \mathrm{kDa}$, significantly heavier than the putative pyruvate transporter. Furthermore, if the two candidate mMCTs were, in fact, the same but fragmented during isolation or electrophoresis, one might find signals at $43 \mathrm{kDa}$ and either 30 or $13 \mathrm{kDa}$. However, that result was not obtained in any of the three isolation procedures used in this study. Hence, the possibility that the variety of MCT1 antibodies used would recognize both a 12-transmembrane MCT1 in the cell membrane and a different 6TM transporter in the mitochondria is unlikely. The protein found in the mitochondrial membrane reported here and earlier is the MCT1 protein.

Both method 1 and method 2 utilize centrifugation steps of 10,000 and $9,000 \mathrm{~g}$, respectively, to pellet mitochondria. These speeds are necessary and appropriate for pelleting mitochondria (19). This is in contrast to method 3, which uses two steps
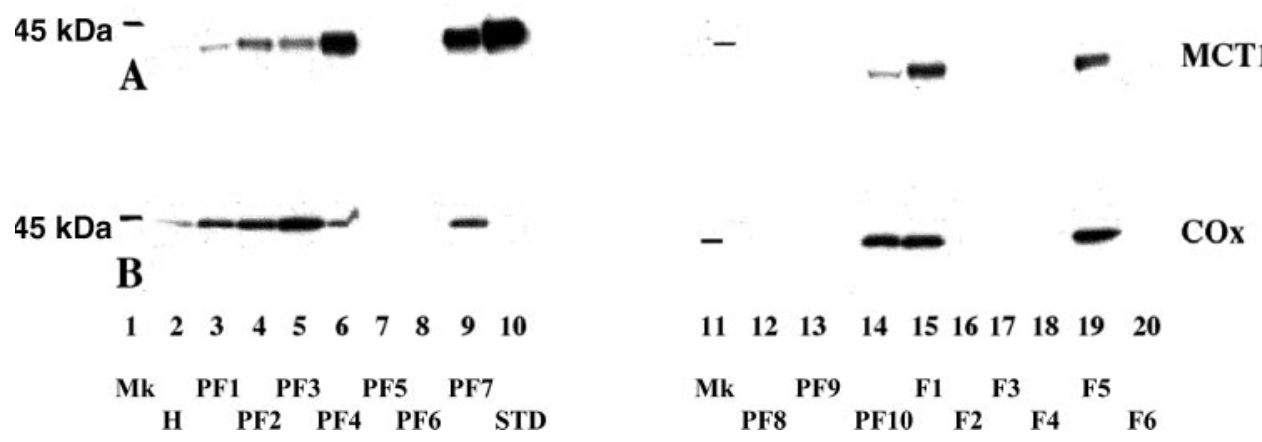

Fig. 7. Multiple Western blots for the existence of MCT1 (left) and cytochrome oxidase (COX; right) in rat skeletal muscle membrane fractions obtained by the procedures of Dombrowski et al. (11) (method 3), used by Bonen et al. (3) and Zhao et al. (31). Lane definitions (as defined by method 3): 1) marker (MK); 2) homogenate (H); 3) protein fraction 1 (PF1); 4) PF2; 5) PF3; 6) PF4; 7) PF5; 8) PF6; 9) PF7; 10) Std; 11) MK; 12) PF8; 13) PF9; 14) PF10; 15) F1; 16) F2; 17) F3; 18) F4; 19) F5; 20) F6. The same membrane is probed with (Brooks) MCT1 antibody $(A)$ and then with COX antibody $(B)$. Note that PF9 (lane 13) represents the L-IM fraction and is not labeled with either MCT1 or COX antibodies. See legend to Fig. 3 and METHODS for details. 
with higher speeds but attempts to look for mitochondrial signals in the resultant supernatant.

The method 3 fractionation procedure used by others to isolate intracellular membranes was developed for studies of GLUT4 translocation. Accordingly, it would appear that contesting mitochondrial localization of MCT1 on the basis of results using the Dombrowski fractionation procedure to isolate mitochondria from other cell fractions as attempted by Zhao et al. (31) is inappropriate. The data presented here indicate that the Dombrowski procedure (method 3) fractionates mitochondria with remnants precipitated out at several steps other than the L-IM (PF9) fraction. The preceding two centrifugation steps occur at 13,000 $g$ and 53,000 $g$ (see Fig. 3), resulting in pellets labeled PF3 and PF4, respectively. These would certainly be expected to pellet any mitochondria. It is interesting to note from COX data presented here (Fig. 7) show that whole mitochondria and mitochondrial fragments are found in many places other than PF9 and that every fraction that contains a signal for $\mathrm{COX}$ also contains a signal for MCT1. Similarly, studies of others (3) using the method of Dombrowski et al. (11) to localize MCT1 in skeletal muscle could not be expected to find mMCT1.

In striated muscle, mitochondrial mass is organized as a reticulum (18), and the isolation of organelles subjects native structures and proteins to numerous degradative processes (e.g., homogenization, proteolytic enzymes, solvents). The use of proteolytic enzymes has been shown to result in loss of labile mitochondrial constituents (4). Care was taken in methods 1 and 2 to isolate mitochondrial fractions without the use of proteolytic enzymes such as nagarse or trypsin, although the homogenization steps were necessarily employed. Use of method 3 either with or without $\mathrm{LiBr}$ resulted in similar relative distributions of MCT1, although the absence of $\mathrm{LiBr}$ was also associated with a reduction of total protein concentrations in all subsequent steps. Regardless of whether the use of $\mathrm{LiBr}$ is necessary for the isolation of GLUT4 vesicles, it does not appear that it is the sole reason for the lack of finding MCT1 in mitochondria.

Data provided with respect to CD147 indicate that it was found only in the MU and SL fractions but not in the MI, SM, or IM fractions (methods 1, 2, and 3, respectively). These data are consistent with the findings of Kirk et al. (17) that CD147 is a chaperone protein, which is associated with the MCT1 and targets the MCT1 to the sarcolemma. Although Kirk et al. also suggested that CD147 is associated with MCT4, acting as a chaperone for its localization to the sarcolemma, MCT4 was not studied here. The lack of CD147 in the mitochondria and the evidence of $\mathrm{mMCT} 1$ presented here and previously support the suggestion that another, different chaperone protein is associated with targeting the MCT1 to the mitochondria.

With the data presented here, mitochondria of rat striated muscle contain MCT1, confirming data previously published (6) but subsequently contested (31). As has been shown previously (7), mitochondria also contain lactate dehydrogenase. This confirms the existence of the machinery necessary to allow the import and oxidation of lactate in the mitochondria. Data published earlier have also demonstrated the ability of mitochondria to respire using lactate as a fuel, and the ability of blocking the transport or lactate dehydrogenase-catalyzed oxidation of lactate also blocks the ability of mitochondria to use lactate as a fuel (7). Existence of this machinery has also been demonstrated in the human mitochondria (12).

Although in this report we have asserted a mitochondrial presence of MCT1, we do not mean to imply sole mitochondrial and sarcolemmal cell domains for MCT1 in striated muscle. To the contrary, ILSs have been demonstrated in the sperm mitochondria in several mammals $(8,13)$. In addition, MCT1 has been localized to liver peroxisomes (21), and a peroxisomal lactate shuttle has been demonstrated to be functional in the rat liver $(1,21)$. Taken together, these data are interpreted to mean that the ILS machinery is present and functional within diverse types of cells.

Part of our strategy for identifying cell domains occupied by MCT1 was based on the report of Kirk et al. (17), who identified CD147 as the sarcolemmal chaperone for MCT1 and MCT4. However, because their focus was on the sarcolemmal as opposed to mitochondrial membranes, the possibility arises that CD147 may be a universal MCT1 chaperone and hence may direct or scaffold MCT1 to the mitochondrial reticulum. Indeed, in Fig. 5 and other autoradiograms developed by us, there is a faint signal for CD147 in the MI fraction. However, at this point, we are inclined to accept the conclusion of Kirk et al. on the basis of relative strengths of MCT1 and CD147 signals in SL and MI fractions. The signal for MCT1 is relatively far stronger that that for CD147 in the MI fraction, whereas signal strengths are alike in SL membrane fractions.

In summary, we have confirmed that MCT1 is localized in both cell membranes and the mitochondria of rat striated muscle. Taken together with previously published data (7), this provides evidence that machinery for both the cell-cell lactate shuttle and the ILS machinery exists as originally proposed (5). These findings are consistent across two different cell fractionation procedures, using both fresh and frozen tissue, and three different MCT1 antibodies, raised against two different hosts. The most likely reason for the previous differences in experimental findings between laboratories seems to be in the experimental procedures used to isolate the subcellular fractions. ${ }^{1}$

\section{ACKNOWLEDGMENTS}

We acknowledge the assistance of Kalle Magnusson for help in the fractionation procedure.

\section{GRANTS}

This investigation was supported by funding from National Institute of Arthritis and Musculoskeletal and Skin Diseases Grant AR-42906. G. B. McClelland was supported by a postdoctoral fellowship from the Natural Sciences and Engineering Research Council of Canada.

\footnotetext{
${ }^{1}$ From our editorial responsibilities, we know that Wenzel et al. have a paper in press showing mitochondrial MCT1 using confocal microscopy in transformed HT-29 incubated colon cells (29a).
}

\section{REFERENCES}

1. Baumgart E, Fahimi HD, Stich A, and Volkl A. L-Lactate dehydrogenase A4- and A3B isoforms are bona fide peroxisomal enzymes in rat liver. Evidence for involvement in intraperoxisomal NADH reoxidation. J Biol Chem 271: 3846-3855, 1996.

2. Bizeau M, Willis W, and Hazel J. Differential responses to endurance training in subsarcolemmal and interfibrillar mitochondria. J Appl Physiol 85: 1279-1284, 1998.

3. Bonen A, Miskovic D, Tonouchi M, Lemieux K, Wilson M, Marette A, and Halestrap A. Abundance and subcellular distribution of MCT1 and 
MCT4 in heart and fast-twitch skeletal muscles. Am J Physiol Endocrinol Metab 278: E1067-E1077, 2000.

4. Brooks GA. Lactate shuttle-between but not within cells? J Physiol 541: 333-334, 2002.

5. Brooks GA. Mammalian fuel utilization during sustained exercise. Сomp Biochem Physiol B Biochem Mol Biol 120: 89-107, 1998.

6. Brooks GA, Brown M, Butz CE, Sicurello JP, and Dubouchaud H. Cardiac and skeletal muscle mitochondria have a monocarboxylate transporter MCT1. J Appl Physiol 87: 1713-1718, 1999.

7. Brooks GA, Dubouchaud H, Brown M, Sicurello JP, and Butz CE. Role of mitochondrial lactic dehydrogenase and lactate oxidation in the intracellular lactate shuttle. Proc Natl Acad Sci USA 96: 1129-1134, 1999.

8. Calvin $\mathbf{J}$ and Tubbs PK. Mitochondrial transport processes and oxidation of NADH by isotonically-treated boar spermatozoa. Eur J Biochem 89: 315-320, 1978.

9. Capuano F, DiPaola M, Azzi A, and Papa S. The monocarboxylate carrier from rat liver mitochondria. Purification and kinetic characterization in a reconstituted system. FEBS Lett 261: 39-42, 1990.

10. Chatham JC, Des Rosiers C, and Forder JR. Evidence of separate pathways for lactate uptake and release by the perfused rat heart. Am J Physiol Endocrinol Metab 281: E794-E802, 2001.

11. Dombrowski L, Roy D, Marcotte B, and Marette A. A new procedure for the isolation of plasma membranes, T tubules, and internal membranes from skeletal muscle. Am J Physiol Endocrinol Metab 270: E667-E676, 1996.

12. Dubouchaud H, Butterfield GE, Wolfel EE, Bergman BC, and Brooks GA. Endurance training, expression and physiology of MCT1 and MCT4 in human skeletal muscle. Am J Physiol Endocrinol Metab 278: E571E579, 2000.

13. Gallina FG, Gerez de Burgos NN, Burgos C, Coronel CE, and Blanco A. The pyruvate/lactate shuttle in spermatozoa: operation in vitro. Arch Biochem Biophys 308: 515-519, 1994.

14. Garcia CK, Brown MS, Pathak RK, and Goldstein JL. cDNA cloning of MCT2, a second monocarboxylate transporter expressed in different cells than MCT1. J Biol Chem 270: 1843-1849, 1995.

15. Garcia CK, Goldstein JL, Pathak RK, Anderson RGW, and Brown MS. Molecular characterization of a membrane transporter for lactate pyruvate, and other monocarboxylates: implication for the Cori cycle. Cell 76: 865-873, 1994.

16. Johannsson E, Lunde PK, Heddle C, Sjaastad I, Thomas MJ, Bergersen L, Halestrap AP, Blackstad TW, Ottersen OP, and Sejersted OM. Upregulation of the cardiac monocarboxylate transporter MCT1 in a rat model of congestive heart failure. Circulation 104: 729-734, 2001.

17. Kirk P, Wilson M, Heddle C, Brown M, Barclay A, and Halestrap A. CD147 is tightly associated with lactate transporters MCT1 and MCT4 and facilitates their cell surface expression. EMBO J 19: 3896-3904, 2000.
18. Kirkwood SP, Munn EA, Packer L, and Brooks GA. Mitochondrial reticulum in limb skeletal muscle. Am J Physiol Cell Physiol 251: C395-C402, 1986.

19. Lehninger AL, Nelson DL, and Cox MM. Principles of Biochemistry. New York: Worth, 1993.

20. McClelland GB and Brooks GA. Changes in MCT1, MCT4 and LDH expression are tissue specific in rat after long-term hypobaric hypoxia. J Appl Physiol 92: 1573-1584, 2002.

21. McClelland GB, Khanna S, Gonzalez GF, Butz CE, and Brooks GA. Peroxisomal membrane monocarboxylate transporters: evidence for a redox shuttle system? Biochem Biophys Res Commun 304: 130-135, 2003.

22. Nalecz M, Nalecz K, Broger C, Bolli R, Wojtczak L, and Azzi A. Extraction, partial purification and functional reconstitution of two mitochondrial carriers transporting keto acids: 2-oxyglutarate and pyruvate. FEBS Lett 196: 331-336, 1986.

23. Pellerin L, Pellegri G, Martin JL, and Magistretti PJ. Expression of monocarboxylate transporter mRNAs in mouse brain: support for a distinct role of lactate as an energy substrate for the neonatal vs. adult brain. Proc Natl Acad Sci USA: 3990-3995, 1998.

24. Poole RC, Sansom CE, and Halestrap AP. Studies of the membrane topology of the rat erythrocyte $\mathrm{H}^{+}$/lactate. Biochem J 320: 817-24, 1996.

25. Price NT, Jackson VN, and Halestrap AP. Cloning and sequencing of four new mammalian monocarboxylate transporter (MCT) homologues confirms the existence of a transporter family with an ancient past. Biochem J 329: 321-328, 1998.

26. Roy D, Johannsson E, Bonen A, and Marette A. Electrical stimulation induces fiber type-specific translocation of GLUT-4 to T tubules in skeletal muscle. Am J Physiol Endocrinol Metab 273: E688-E694, 1997.

27. Roy D and Marette A. Exercise induces the translocation of GLUT4 to transverse tubules from an intracellular pool in rat skeletal muscle. Biochem Biophys Res Commun 223: 147-152, 1996.

28. Schwoch G and Passow H. Preparation and properties of human erythrocyte ghosts. Mol Cell Biochem 2: 197-218, 1973.

29. Valenti D, de Bari L, Atlante A, and Passarella S. L-Lactate transport into rat heart mitochondria and reconstruction of the L-lactate/pyruvate shuttle. Biochem J 364: 101-104, 2002.

29a.Wenzel U, Schoberl K, Lohner K, and Daniel H. Activation of mitochondrial lactate uptake by flavone induces apoptosis in human colon cancer cells. J Cell Physiol In press.

30. Yoon H, Fanelli A, Grollman EF, and Philip NJ. Identification of a unique monocarboxylate transporter (MCT3) in retinal pigment epithelium. Biochem Biophys Res Commun 234: 90-94, 1997.

31. Zhao C, Wilson M, Schuit F, Halestrap AP, and Rutter G. Expression and distribution of lactate/monocarboxylate transporter isoforms in pancreatic islets and the exocrine pancreas. Diabetes 50: 361-366, 2001. 